\title{
An Equivalent Noise Investigation of Saccadic Suppression
}

\author{
Tamara Watson ${ }^{1,2}$ and Bart Krekelberg ${ }^{1}$ \\ ${ }^{1}$ Center for Molecular and Behavioral Neuroscience, Rutgers University, Newark, New Jersey 07102 and ${ }^{2}$ School of Psychology, University of Western \\ Sydney, Penrith, New South Wales 2751, Australia
}

Visual stimuli presented just before or during an eye movement are more difficult to detect than those same visual stimuli presented during fixation. This laboratory phenomenon- behavioral saccadic suppression-is thought to underlie the everyday experience of not perceiving the motion created by our own eye movements-saccadic omission. At the neural level, many cortical and subcortical areas respond differently to perisaccadic visual stimuli than to stimuli presented during fixation. Those neural response changes, however, are complex and the link to the behavioral phenomena of reduced detectability remains tentative. We used a well established model of human visual detection performance to provide a quantitative description of behavioral saccadic suppression and thereby allow a more focused search for its neural mechanisms.

We used an equivalent noise method to distinguish between three mechanisms that could underlie saccadic suppression. The first hypothesized mechanism reduces the gain of the visual system, the second increases internal noise levels in a stimulus-dependent manner, and the third increases stimulus uncertainty. All three mechanisms predict that perisaccadic stimuli should be more difficult to detect, but each mechanism predicts a unique pattern of detectability as a function of the amount of external noise.

Our experimental finding was that saccades increased detection thresholds at low external noise, but had little influence on thresholds at high levels of external noise. A formal analysis of these data in the equivalent noise analysis framework showed that the most parsimonious mechanism underlying saccadic suppression is a stimulus-independent reduction in response gain.

\section{Introduction}

From the perspective of maintaining perceptual stability, saccadic eye movements are thought of as a necessary evil. Due to the composition of the retina, only the small region of the visual scene viewed by the fovea is observed at high-spatial resolution at any one time. Saccades are necessary to move the fovea around the visual scene, thereby building up a clear picture of the whole scene. The act of moving the eyes, although necessary for the clear picture of the world we perceive, threatens perceptual stability because it creates high-velocity motion across the retina. Subjectively, we do not perceive this perisaccadic motion even when making several eye movements per second. This subjective lack of awareness of the visual consequences of a saccade is termed saccadic omission (2008).

Saccadic omission likely results from a number of different, interrelated components, but we focus here on active saccadic suppression, which we define operationally as the reduced visibility of stimuli flashed on an otherwise blank screen before the eye starts to move (Burr et al., 1982, 1994). This particular configuration has two advantages. First, the retinal input can be made identical to that of stimuli presented during fixation. Sec-

Received Nov. 30, 2010; revised Feb. 24, 2011; accepted March 16, 2011.

Author contributions: T.W. and B.K. designed research; T.W. performed research; T.W. and B.K. analyzed data; T.W. and B.K. wrote the paper.

This work was supported by the Human Frontiers Science Program (T.W.), the National Eye Institute (Grant R01EY17605), and the Pew Charitable Trusts (B.K.).

Correspondence should be addressed to Tamara Watson, School of Psychology, University of Western Sydney, Locked Bag 1797, Penrith South DC, NSW 1797, Australia. E-mail: t.watson@uws.edu.au.

DOI:10.1523/JNEUROSCI.6255-10.2011

Copyright $@ 2011$ the authors $\quad 0270-6474 / 11 / 316535-07 \$ 15.00 / 0$ ond, the reduction of visibility under these circumstances is known to require the actual execution of the saccade; visibility is reduced considerably less when the (unstructured) background is moved in a way that simulates the retinal motion created by the saccade (Diamond et al., 2000). Hence, these two aspects allow us to isolate and study what has been called the active component of saccadic omission (Wurtz, 2008).

Conceptually, saccadic suppression is thought to reflect the influence of a corollary signal from eye movement control areas on visual areas of the brain. Our goal here is to distinguish among three main theories concerning how the corollary discharge signal achieves saccadic suppression. The first stipulates that a reduction in visual response gain causes an increased threshold (Burr et al., 1994). The second claims that a brief increase in internal noise swamps the visual signal and leads to poor detection performance (Diamond et al., 2000). The third states that detection is poor because temporary uncertainty about the location of the stimulus reduces sensitivity (Greenhouse and Cohn, 1991; Binda et al., 2009). We will refer to these as the gain reduction, noise injection, and uncertainty theories, respectively.

Because all three mechanisms may play a role in saccadic suppression, we adapted the equivalent noise framework developed by $\mathrm{Lu}$ and Dosher (1998) that encompasses all three of these mechanisms (see Fig. 2). In this framework, the inner workings of a detection mechanism are revealed by investigating how detection performance changes when the target stimulus is embedded in visual noise. Specifically, we measured contrast detection thresholds for stimuli embedded in Gaussian noise presented at fixation or presaccadically, and found a distinct pattern that was most parsimoniously explained by assuming only a presaccadic 
a

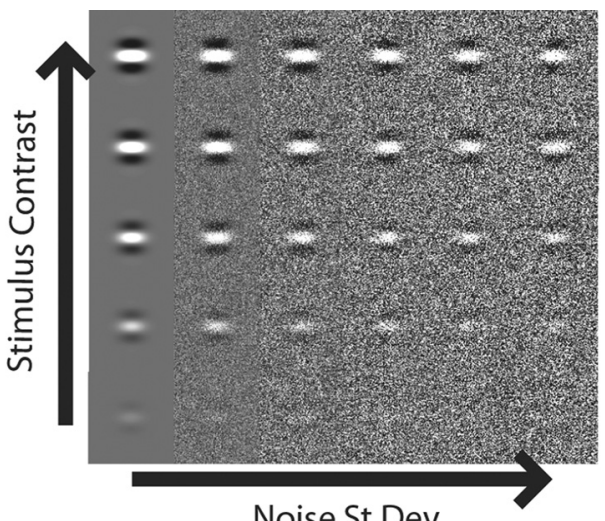

b

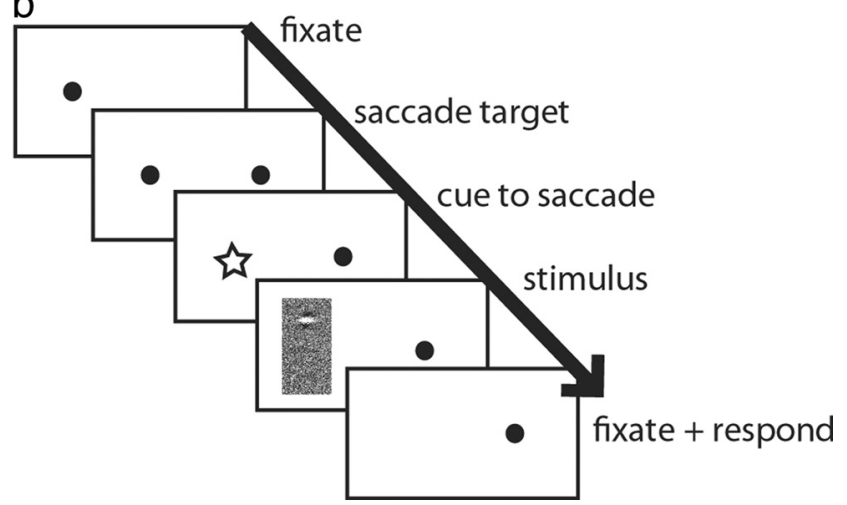

Figure 1. Stimuli and experimental procedure. $\boldsymbol{a}$, The target stimuli were horizontal sine wave gratings at five levels of contrast (vertical axis). The grating edges were smoothed with a Gaussian window. Each stimulus pattern could appear in one of six levels of Gaussian noise (horizontal axis). $\boldsymbol{b}$, Sequence of events during a single trial. Solid points represent the fixation and saccade target. The star denotes the offset of the fixation point (and the cue to make the saccade). St Dev, Standard deviation.

reduction in gain. Presaccadic changes in internal noise or uncertainty did not contribute significantly to saccadic suppression. This suggests that active saccadic suppression of presaccadic stimuli relies on a stimulus-independent reduction in gain of the visual system.

\section{Materials and Methods}

\section{Subjects}

Four subjects (three male) performed the experiments. The three naive participants received remuneration and all had normal or corrected-tonormal vision. The experiments were in agreement with the protection of human subjects as described in the declaration of Helsinki and approved by the Rutgers University institutional review board.

\section{Stimulus}

The target stimulus was a horizontal sine wave grating subtending $4 \times 4$ degrees of visual angle (dva) with the outer edges smoothed by a Gaussian window. The grating had a spatial frequency of 0.3 cycles per degree and a mean luminance of $38 \mathrm{~cd} / \mathrm{m}^{2}$. On separate trials, the Michelson contrast of the grating was of $2,8,16,24$, or $32 \%$. The grating could be presented 4 dva above or below fixation (see Procedure, below).

The target stimulus was embedded in a rectangle of Gaussian noise subtending 4 by 12 dva and centered on the fixation point (Fig. 1). On separate trials, the SD of the noise was $0,2,8,16,24$, or $32 \%$. Both the target stimulus and the Gaussian noise were presented for $16 \mathrm{~ms}$. The background was a constant gray at $38 \mathrm{~cd} / \mathrm{m}^{2}$ and the fixation point was a small white square.

\section{Apparatus}

We generated visual stimuli with Neurostim (http://neurostim.sourceforge. net) and presented them on a Sony FD Trinitron (GDM-C520) CRT monitor with a resolution of $1024 \times 768$ pixels and a refresh rate of 120 $\mathrm{Hz}$. The pupil of the left eye was tracked at a sample rate of $500 \mathrm{~Hz}$ and a nominal spatial resolution of 0.1 dva (Eyelink II; SR Research). Participants sat $57 \mathrm{~cm}$ from the display in a darkened room. Individually molded bite bars restricted their head movements.

\section{Procedure}

We randomly interleaved blocks of saccade and fixation conditions. Trials in which the subject's eyes strayed outside a virtual $2^{\circ} \times 2^{\circ}$ fixation window were terminated and discarded. The terminated trial was repeated at a later stage within the same test session. Each subject completed 1500 successful trials for both the saccade and fixation conditions. At fixation, 300 trials were presented per block for a total of five blocks. Given the six noise contrast levels and five signal contrast levels, each condition occurred 10 times in one session and a total of 50 times. During presaccadic presentation, the total numbers of trials in each condition were governed by the identity of the trials that were discarded due to incorrect fixation. Participants completed 50 or more trials in each condition.

Fixation condition. Trials started with the appearance of a fixation dot at the center of the screen; subjects fixated the dot and the stimulus appeared $1 \mathrm{~s}$ later. Subjects used a standard keyboard to indicate whether the grating target appeared above or below the fixation point. After the response, the fixation dot disappeared and the screen remained blank for $1.5 \mathrm{~s}$ until the next trial began. There were equal numbers of trials with the target above and below fixation.

Saccade condition. The saccade conditions contained two fixation points: an initial fixation and a saccade target (Fig. 1). The initial fixation was presented $7^{\circ}$ to the left of the midpoint of the monitor and the saccade target was presented $7^{\circ}$ to the right of the midpoint of the monitor.

Participants first fixated on the initial fixation dot; $500 \mathrm{~ms}$ after fixation was achieved, the saccade target appeared. The cue to move the eyes (disappearance of the initial fixation point) was given $1975 \mathrm{~ms}$ after initial fixation. Participants were required to fixate on the saccade target within $2250 \mathrm{~ms}$ after initial fixation. Between $2110-2135 \mathrm{~ms}$ after initial fixation, the noise plus target stimulus appeared centered around the location of the now invisible initial fixation point. This timing was adjusted for each participant to present most stimuli within the window of saccadic suppression but before the eye had started to move. Only trials where the stimulus onset actually occurred between 50 and $16 \mathrm{~ms}$ before onset of the saccade were included in the analysis. This window was chosen to maximize the influence of saccadic suppression (Diamond et al., 2000) while ensuring that the stimulus was presented to a stationary retina. Participants reported the location of the grating target via a key press after completion of the eye movement. Registration of a response initiated the next trial.

\section{Analysis}

For each psychometric curve- describing the fraction of correct responses as a function of grating contrast-we determined the best-fitting Weibull function, using the psignifit toolbox (Wichmann and Hill, 2001) in Matlab (Mathworks). We defined the subject's threshold as the grating contrast at which the performance reached a $d^{\prime}$ of 2 ( $85 \%$ correct).

Detection model. We first introduce the detection model that we used to investigate saccadic suppression. The model is based on the noisy perceptual template model (PTM) that Lu and Dosher (1998) used to investigate attentional mechanisms. We present details here to clarify its interpretation in the context of saccadic suppression.

In a typical saccadic suppression experiment, a target is briefly flashed either above or below the fixation point (Fig. $2 a$ ) and the observer reports where the target was located in a two-alternative forced-choice paradigm. We model this decision process by first assuming that there are two templates that are tuned for the target stimulus (Fig. 2b). One of these templates monitors the top location, the other the bottom location. One can think of the template as a population of neurons that are tuned to the spatial frequency and orientation of the target stimulus, and whose response increases proportionally with the contrast of the target. Note, however, that the implementation of the template is irrelevant to our 


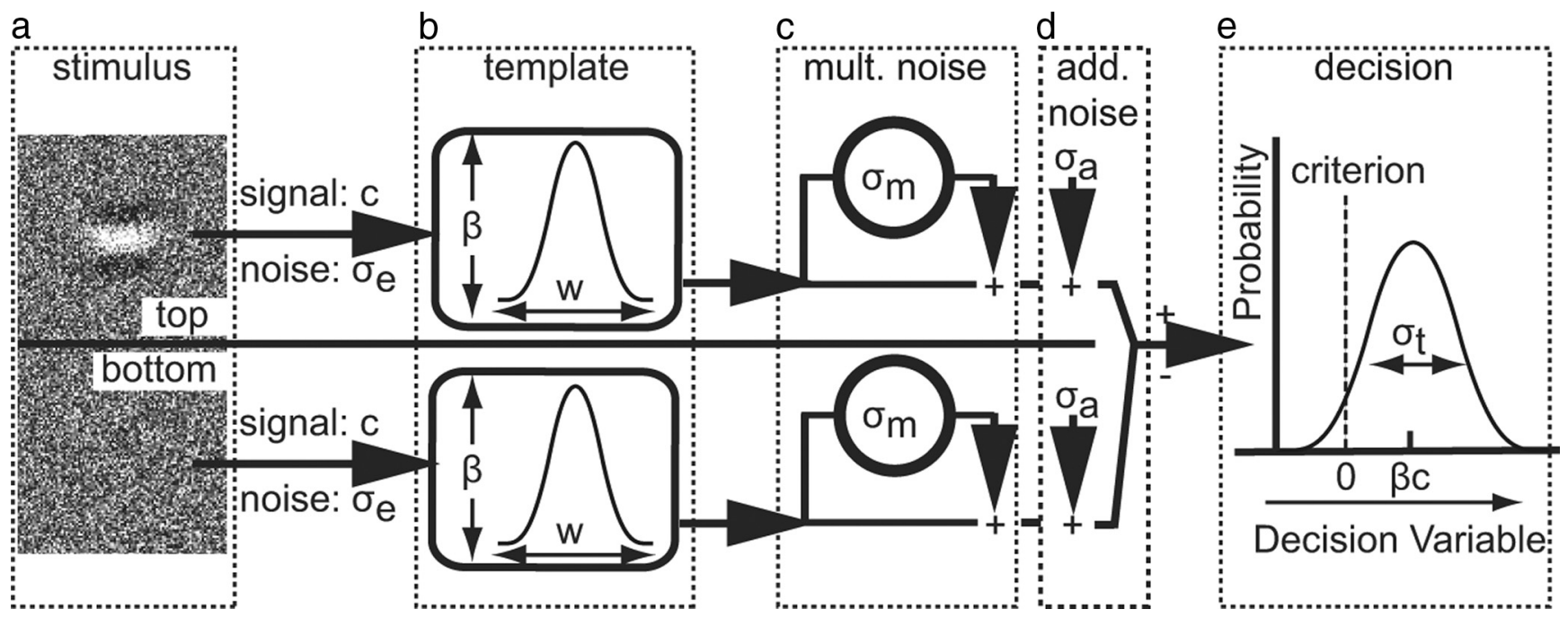

Figure 2. The perceptual template model. $\boldsymbol{a}$, A grating stimulus is embedded in external visual noise. The detector's task is to determine whether the signal is in the top or bottom location. $\boldsymbol{b}$, The template detects the signal and amplifies both signal and noise with gain $\beta$. Selective filtering of noise is represented by the width of the template ( $w$ ); a narrow template passes less of the external noise. $\boldsymbol{c}$, The internal signal is corrupted by multiplicative (mult.) noise, which scales with the power of the output of the template. The amount of noise is specified with the free parameter $\sigma_{m}$. $\boldsymbol{d}$ The internal signal is further corrupted by a fixed amount of additive (add.) noise $\left(\sigma_{a}=1\right)$. An equivalent detector monitors the lower part of the visual field, which contains only noise in this example. $\boldsymbol{e}$, An internal DV is calculated as the difference between the top and bottom detector. Assuming that all sources of noise are independent and Gaussian, the distribution of DV over all trials in which the stimulus was presented at the top is a Gaussian with a SD $\left(\sigma_{t}\right)$ that represents the combination of all noise sources. To reach a decision on a given trial, the model compares the decision variable against a criterion value of zero. If DV $>0$, the model decides "top;" if DV $<0$, the model decides "bottom" (Lu and Dosher, 1998).

current purpose as long as the output of the template is proportional to the contrast of the target. Formally, the template provides a signal $(S)$ to the internal decision mechanism that is proportional to the contrast $(c)$ of the input, $S=\beta c$, with $\beta$ a gain parameter. Because only a single target is presented on each trial, $c$ will equal 0 for the template that does not receive the target (Fig. $2 b$, bottom). In the absence of all internal and external noise, the simple decision rule to choose top if $\beta c>0$ and bottom if $\beta c<0$ would lead to perfect performance. The PTM, however, has three stages at which noise is introduced. The following paragraphs outline the progressive corruption (increase in variance) of the decision signal in the PTM.

The first source of noise is the Gaussian external visual noise that was presented together with the stimulus (defined by its SD $\sigma_{e}$ ) (Fig. $2 a$ ). Both the gain and the width of the perceptual template (Fig. $2 b$ ) affect the influence of the external noise on the internal noise. The gain simply scales the external noise, just as it scales the signal. A narrow template corresponds to a detection mechanism that knows where in space to look for the target - the mechanism is spatially selective. A broad template, in contrast, sums signal (and noise) across a large range of space; it is spatially uncertain. Mathematically, we implement this by simply multiplying the SD of the external noise by a factor that corresponds to the width $(w)$ of the template. If the template is wide (i.e., $w$ is larger), the effect of the noise is stronger (larger SD). With these definitions, the output of the template is the random variable $\beta\left(c+w N\left(0, \sigma_{e}\right)\right)$, where $N(\mu, \sigma)$ represents a Gaussian random variable with mean $\mu$ and SD $\sigma$. Hence, after the template stage (Fig. $2 b$ ), the mean of the internal signal is $\beta c$ and its variance is $\left(\beta \mathrm{w} \sigma_{e}\right)^{2}$.

The second source of noise is the multiplicative internal Gaussian noise that acts on the output of the perceptual template (Fig. $2 c$ ). The PTM assumes that the average contrast (power) of the template determines the strength of the multiplicative noise. Because the power of the output of the template is $\beta^{2}\left(c^{2}+\left(\mathrm{w} \sigma_{e}\right)^{2}\right)$, the internal multiplicative noise source adds noise to the internal signal with a variance equal to $\sigma_{m}^{2} \beta^{2}\left(c^{2}+\left(w \sigma_{e}\right)^{2}\right)$, where $\sigma_{m}$ represents the free parameter we used to describe the strength of the internal multiplicative noise. Note that the variance contributed by this noise source scales with both signal (c) and the fraction of the external noise that is passed through the template $\left(w \sigma_{e}\right)$.

The third source of noise in the model is additive independent Gaussian noise with SD $\sigma_{a}$ (Fig. $2 d$ ). We did not vary this parameter in our model because its influence on contrast thresholds is formally equivalent to the template gain parameter $\beta$ (Eq. 3) (see Discussion, below). Hence, this noise source adds a constant $\sigma_{a}^{2}$ to the variance of the internal signal.

At the output stage of the detector, the signal of the top and bottom detectors are subtracted to generate the decision variable (DV) (Fig. $2 e$ ). In the absence of noise, DV would be $\beta c$ for any trial in which a stimulus of contrast $c$ was presented to the top detector only. The presence of external and internal noise, however, makes DV a random variable. Because all noise sources have zero mean, they do not affect the mean of DV. Moreover, because all noise sources are independent and Gaussian distributed, their total variance $\left(\sigma_{t}^{2}\right)$ equals the sum of all variances, as follows:

$$
\begin{aligned}
\sigma_{t}^{2}=\left[\left(\beta w \sigma_{e}\right)^{2}+\sigma_{m}^{2} \beta^{2}\left(c^{2}+\left(w \sigma_{e}\right)^{2}\right)+\right. & \left.\sigma_{a}^{2}\right]+\left[\left(\beta w \sigma_{e}\right)^{2}\right. \\
& \left.+\sigma_{m}^{2} \beta^{2}\left(w \sigma_{e}\right)^{2}+\sigma_{a}^{2}\right] .
\end{aligned}
$$

The brackets group the variance from the detector with signal and without signal, and the terms within the bracket correspond to the noise introduced at each stage of the detector (template, multiplicative, additive). In other words, the decision variable DV is a Gaussian random variable with mean $\beta c$ and SD $\sigma_{t}$. Figure $2 e$ shows this distribution of the decision variable over many trials in which a stimulus with contrast $c$ was presented in the top location. An ideal observer following the decision rule to state "top" when DV $>0$ will have a performance $(d$ ') given by the following:

$$
d^{\prime}=\frac{s}{\sigma_{t}}=\frac{\beta c}{\sqrt{2\left(\beta w \sigma_{e}\right)^{2}+\sigma_{m}^{2} \beta^{2}\left(c^{2}+2\left(w \sigma_{e}\right)^{2}\right)+2 \sigma_{a}^{2}}} .
$$

This equation can be solved to give the contrast threshold for a given level of performance $\left(d^{\prime}\right)$ as:

$$
c=\sqrt{2 \frac{\left(w \sigma_{e}\right)^{2}\left(1+\sigma_{m}^{2}\right)+\frac{\sigma_{a}^{2}}{\beta^{2}}}{\left(\frac{1}{d^{\prime 2}}-\sigma_{m}^{2}\right)} .}
$$

This formula describes how threshold contrast $(c)$ depends on the external noise $\left(\sigma_{e}\right)$ for a given performance $\left(d^{\prime}\right)$. Each of the free parameters in 
this model (template gain, $\beta$; template width, $w$; and multiplicative noise, $\sigma_{m}$ ) plays a unique role in shaping this curve. Next, we will discuss these three roles and link them to specific theories about saccadic suppression.

Models of saccadic suppression in the equivalent noise framework. Decreasing the gain $(\beta)$ of the template will reduce performance at low external noise, but because the gain is applied to noise and signal, the influence of a gain change at high external noise is minimal. This functional dependence is shown in Figure $3 a$. The gain reduction theory of saccadic suppression suggests that the gain of the visual response is reduced early in the processing hierarchy (Burr et al., 1994). As a consequence, subsequent visual centers receive a weaker visual signal, and therefore detection thresholds increase. Hence, in terms of the detection model, this theory predicts a perisaccadic reduction of the $\beta$ parameter and therefore a change in threshold as a function of external noise between fixation and perisaccadic presentation (Fig. 3a).

Increasing the noise parameter $\sigma_{m}$ of the model makes a very different prediction. Because this internal noise is multiplicative, the total internal noise increases proportional to the external noise, and thresholds change (Fig. 3b). This model parameter manipulation maps onto the noise injection theory of saccadic suppression, which suggests that a brief spike of activity is injected into the visual system (Diamond et al., 2000). There are two variants of this theory. In the first, the injected noise is stimulus independent; this model is formally equivalent to the gain reduction model and will not be considered separately here (see Discussion, below). In the second variant, which we do consider, the injected noise is proportional to the input; this corresponds to a perisaccadic increase in the multiplicative noise $\sigma_{m}$. This theory predicts experimental performance (Fig. 3b).

The width of the template $(w)$ does not affect performance at low levels of external noise where the signal dominates the response. At high levels of external noise $\left(\sigma_{e}\right)$, however, a wider template will be dominated by the noise, and hence increase thresholds (Fig. $3 c$ ). This model parameter manipulation maps onto the uncertainty theory of saccadic suppression, which postulates that the location of a stimulus is uncertain around an eye movement; either because the eye moves while the detection is ongoing (Greenhouse and Cohn, 1991), because the internal eye-position signal is not updated with high fidelity, or because receptive fields shift their location (Binda et al., 2009). Regardless of the source of uncertainty, visual detection thresholds are expected to suffer because the signal that is to be detected can now appear in multiple locations. When integrating over those multiple locations, more noise enters into the decision process and detection thresholds increase. In our detection model, this corresponds to a perisaccadic increase in the template width $(w)$. Therefore, this theory predicts experimental performance (Fig. $3 c$ ).

Our experiments tested these qualitatively different predictions about changes in threshold as a function of external noise.

Model fitting. We used a nonlinear least-squares curve-fitting algorithm (lsqcurvefit in Matlab) to fit the parameters of the closed form of the perceptual template model (Eq. 1) to the individual subject threshold data.

We fitted all possible combinations of constrained models in which one or more of the parameters were set to a neutral value (e.g., $\sigma_{m}=0$, $w=1$, to fit a model in which only $\beta$ was a free parameter). To select the most parsimonious model, we calculated the Akaike information criterion (AIC) (Akaike, 1974) for each constrained model. Low AIC values correspond to more parsimonious models. By convention, if the difference in AIC values of two models is between 0 and 2, there is still substantial support for the less parsimonious model, but if the difference is between 4 and 7, the support for the model with the higher AIC is considerably less. Models that have AIC values that are $>10$ larger than the
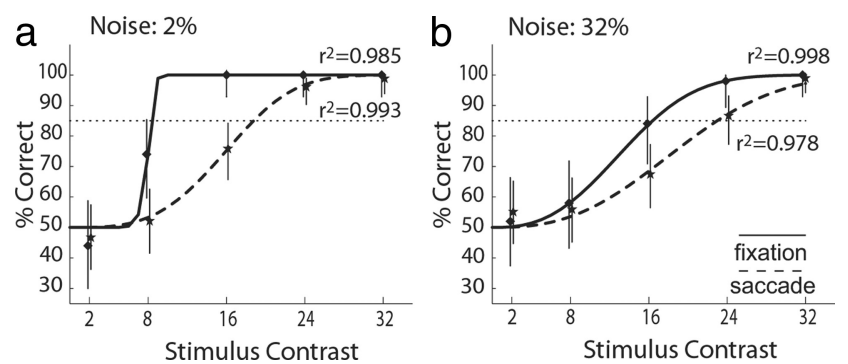

Figure 4. Single subject threshold estimation. The curves show the percentage of correctly localized gratings as a function of stimulus contrast. Diamonds, Performance during fixation; stars, performance for grating stimuli presented just before a saccade. The lines represent Weibull fits to the data during fixation (solid) or just before saccades (dashed). Error bars represent $95 \%$ confidence intervals. $\boldsymbol{a}$, Performance at low (2\%) external noise. $\boldsymbol{b}$, Performance at high (32\%) external noise. These data show that impending saccades reduce detection performance, but less so in the presence of strong external noise.

best model have essentially no empirical support (Burnham and Anderson, 2002).

\section{Results}

\section{Experimental results}

The model simulations in Figure 3 demonstrate that the way in which contrast thresholds change with external noise provides a strong constraint on the parameters of the detection model. Hence, we measured contrast thresholds for gratings embedded in varying levels of external noise. In brief, sine wave gratings, embedded in noise, were presented either above or below the fixation point, and either during steady fixation or just before a saccade. Subjects reported the location of the target stimulus.

Figure 4 shows psychometric curves for a single subject at a low and high level of external noise, for stimuli presented at fixation, and presaccadically. The impending saccade had a large influence on the performance at low levels of external noise (Fig. $4 a$ ), but only a small effect on threshold at high levels of external noise (Fig. 4b). To quantify this, we fitted Weibull functions to each psychometric curve and determined the threshold (across subjects, all perisaccadic Weibull fits had $R^{2}>0.9$ with a mean $R^{2}$ of 0.98 , and all fixation Weibull had $R^{2}>0.92$ with a mean $R^{2}$ of 0.98). Figure 5 plots these thresholds as a function of the external noise. Figure $5 c$ shows the results for the subject whose raw data 

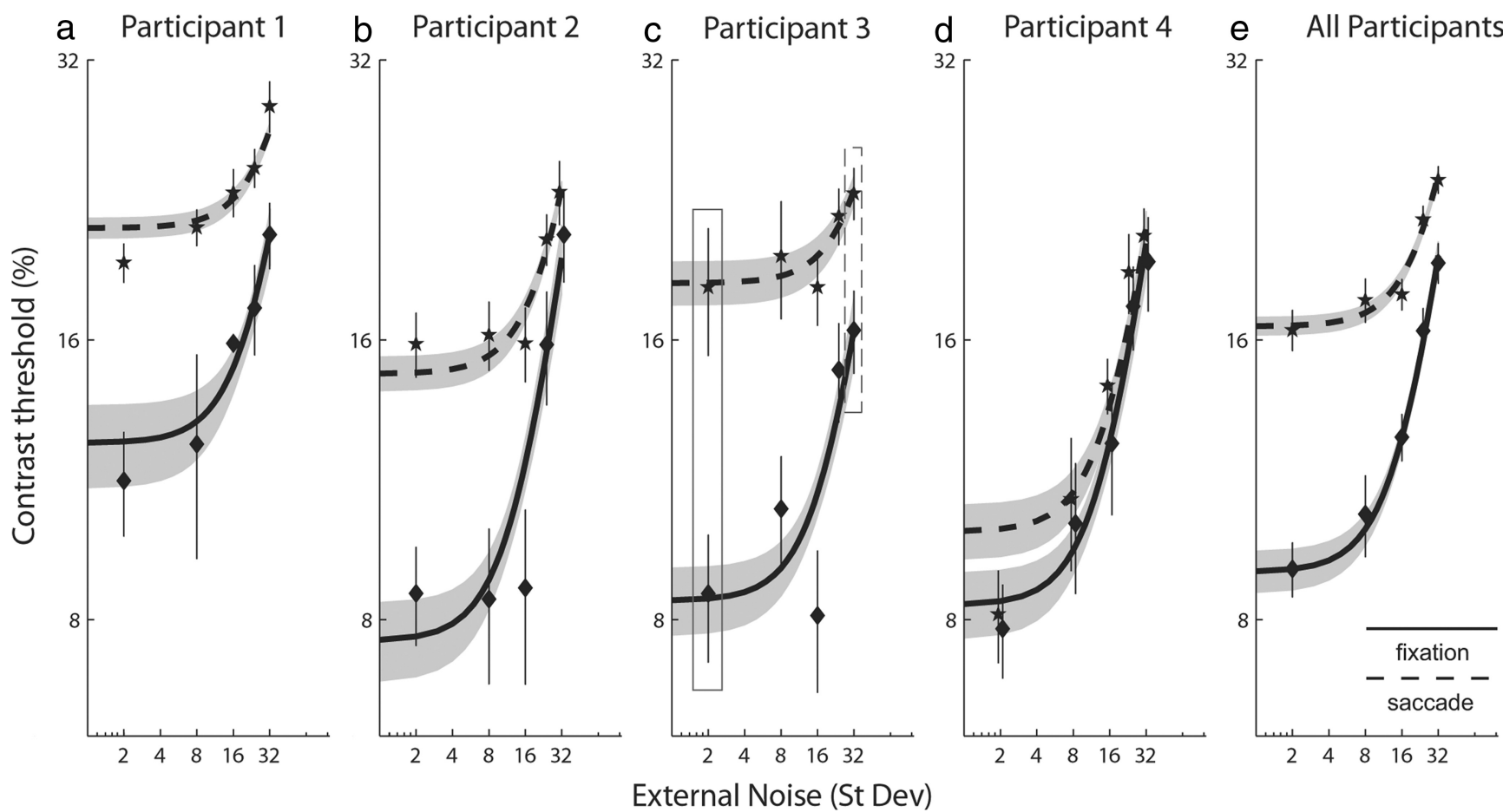

Figure 5. Changes in detection thresholds with external noise level. $\boldsymbol{a}-\boldsymbol{d}$, Results for four individual participants. $\boldsymbol{c}$, Boxes, Thresholds obtained from data in Figure 4; solid line, threshold from Figure $4 a$; dashed line, threshold from Figure $4 b . \boldsymbol{e}$, The average of all participants. $\boldsymbol{a}-\boldsymbol{e}$, Diamonds, thresholds at fixation; stars, thresholds for stimuli presented just before a saccade. Error bars represent 2 SDs of the threshold values estimated via bootstrapping. Solid lines, Best fitting, most parsimonious perceptual template model for fixation; dashed line, best fitting, most parsimonious perceptual template model for saccade conditions; shading, 2 SDs of the fits obtained via bootstrapping. These data show that contrast thresholds converge at high levels of external noise; this is consistent with a gain reduction model. St Dev, Standard deviation.

are shown in Figure 4; the boxes indicate the thresholds shown in Figure $4, a$ and $b$, respectively.

Figure 5 confirms that participants' thresholds were consistently lower at fixation than presaccadically. This corroborates the well established fact that low spatial frequency gratings are less visible presaccadically than during steady fixation. The pattern of results as external noise level increased was consistent across participants. Notably, the fixation and perisaccadic thresholds converged as the external noise level increased. This implies that the mechanism that underlies this form of saccadic suppression becomes ineffective at high levels of external noise.

Comparing these curves with the model predictions in Figure 3 qualitatively suggests a model relying on a presaccadic gain change. In the following paragraphs, we perform quantitative model fits and model selection to support this claim.

\section{Model fits}

We used a nonlinear least-squares fit to estimate model parameters based on the data shown in Figure 5. We repeated this calculation for all constrained models-models in which one or more of the parameters was set to a neutral value (i.e., all combinations of $\beta=1, w=1$, or $\sigma_{m}=0$ ). For each of these model fits, we calculated the Akaike information criterion (Akaike, 1974) to determine the most parsimonious model.

At fixation, the best model included template gain and width as free parameters (AIC for model fit to average of all participant's data $=-50.72$ ). Allowing the multiplicative noise to vary increased the AIC value by 23 , thus strongly arguing that the model with only gain and width as free parameters was a parsimonious description of the data.

Using the best fitting fixation model (per subject) as the starting point, we next determined which parameters in the model
Table 1. Parameter values and goodness of fit for the best model at fixation and prior to a saccade

\begin{tabular}{lllllll}
\hline & \multicolumn{2}{l}{ Fixation } & & & \multicolumn{2}{l}{ Saccade } \\
\cline { 2 - 3 } Participant & $\begin{array}{l}\text { Template } \\
\text { gain }(\beta)\end{array}$ & $\begin{array}{l}\text { Template } \\
\text { width }(w)\end{array}$ & $\begin{array}{l}\text { Explained } \\
\text { variance }(\%)\end{array}$ & & $\begin{array}{l}\text { Template } \\
\text { gain }(\beta)\end{array}$ & $\begin{array}{l}\text { Explained } \\
\text { variance }(\%)\end{array}$ \\
\hline 1 & 24 & 0.18 & 93 & 14 & 93 \\
2 & 38 & 0.19 & 91 & & 20 & 92 \\
3 & 35 & 0.15 & 82 & & 16 & 82 \\
4 & 35 & 0.20 & 97 & & 29 & 94 \\
Average & 32 & 0.19 & 99 & & 18 & 97 \\
\hline
\end{tabular}

The additive noise in all models was fixed to 1 . Because there was no empirical support for a role of the multiplicative noise parameter, its value was 0 in all models.

should be varied to provide the most parsimonious account of the presaccadic thresholds. Consistent with the qualitative match between the data and Figure $3 a$, only a decrease in gain was needed to fit the data in each of the subjects (AIC for model fit to average of all participant's data $=-56.24$ ). The difference in AIC value with any of the other, more elaborate models was at least 10, hence there is strong support for the claim that the data only require a gain change between fixation and saccade conditions. The estimated model parameters for each of the subjects are shown in Table 1. On average, the presaccadic gain was $60 \%$ of the fixation gain.

For completeness, we also investigated models in which the saccadic fits were not constrained by the fixation data. The results remained the same: template gain and width explained the data most parsimoniously, and only the gain changed between fixation and saccade conditions.

\section{Discussion}

We used an equivalent noise analysis method to determine the mechanism responsible for the reduction in contrast sensitivity 
of stimuli presented just before a saccade. Detection thresholds were increased presaccadically, but this saccadic suppression decreased as the stimuli were embedded in increasing Gaussian noise. This pattern of results is a signature of a gain-reduction mechanism. We quantitatively confirmed that a model assuming a presaccadic drop in response gain parsimoniously accounted for the change in contrast detection thresholds. There were two other competing explanations: the noise injection and the uncertainty models. The essence of the multiplicative noise injection model is that to hide any visual input during a saccade, the system generates an amount of internal noise that is proportional to that input. Our data show that this does not play an important role in active saccadic suppression. The spatial uncertainty mechanism explains saccadic suppression as the consequence of an increase in spatial uncertainty and concomitant increase in noise around saccades; our data do not provide support for this mechanism.

\section{Mechanisms}

In the perceptual template model, a decrease in template gain is formally equivalent to an increase in additive internal noise ( $\mathrm{Lu}$ and Dosher, 1998). The reason for this is that in this model, only the signal-to-noise ratio at the output of the detector matters; hence, a lower gain has the same effect as additive noise. A formal proof of this can be seen in Equation 3, where only the ratio of additive noise $\sigma_{a}$ and gain $\beta$ plays a role. Hence, a decrease in gain is equivalent to an increase in additive noise. Because of this formal parameter equivalence, an equally valid interpretation of our results is that saccadic suppression involves the injection of additive (i.e., input-independent) noise. Diamond et al.'s (2000) model falls in this category; they postulated that corollary discharge leads to a stimulus-independent, transient increase in activity across all visual detectors. In other words, their model assumed that additive noise was injected into the visual system. Our analysis suggests that the same results could have been obtained by assuming a transient decrease in gain. Whether additive noise or a reduction in gain is a better description of the neural implementation is something that our behavioral methods cannot address. Neural and functional imaging data discussed below, however, suggest that a combination of these mechanisms may operate in the brain.

Given the similarity in name, it is important to point out that a gain change in the perceptual template model is qualitatively different from contrast gain control-a mechanism whose role is well established in visual processing (Heeger, 1992; Carandini and Heeger, 1994; Carandini et al., 1997). In fact, contrast gain control is stimulus-dependent and analogous to a change in the multiplicative noise parameter ( $\mathrm{Lu}$ and Dosher, 1998). Because we find no evidence for a presaccadic change in multiplicative noise, our data suggest that active saccadic suppression does not involve contrast gain-control mechanisms.

Our findings show that perisaccadic increases in contrast thresholds are not caused by perisaccadic increases in spatial uncertainty. This result may appear at odds with Greenhouse and Cohn (1991), who reported a reduced slope of receiver operating characteristics curves during saccades compared with at fixation. Such reduced slopes can be the consequence of an increase in uncertainty. A likely explanation for the differing results is the timing of the stimuli. Greenhouse and Cohn (1991) presented the target stimulus after the eye had begun to move. This implies that the target position was necessarily uncertain (and possibly smeared out) in the retinal coordinates on which the early visual system relies. Hence, the uncertainty mechanism identified by Greenhouse and Cohn (1991) appears to be an example of a passive mechanism: the inevitable consequence of presenting stimuli to moving eyes is that its location is uncertain. This uncertainty contributes to saccadic omission, but our focus here was on active mechanisms of saccadic suppression. Our data suggest that active saccadic suppression does not involve an increase in uncertainty.

In their perceptual template model, Lu and Dosher (1998) allowed the multiplicative noise to vary as a power law function of the output of the template. This introduces one extra free parameter in the model ( $\gamma$, the power). In the experiments of $\mathrm{Lu}$ and Dosher (1998), the value of this parameter was larger than one. We investigated this possibility in our dataset, but found no experimental support for a power different from one. Given that the multiplicative noise term in itself did not contribute significantly to our model fits, this should not be surprising. The lack of multiplicative noise effects in our data may be related to the task [our subjects performed a contrast detection task, whereas Lu and Dosher (1998) used a more complex task involving orientation discrimination]. This leads to the natural follow-up idea that, given a different task, the internal signal processing could be more complex, and this processing could also be modulated differently by an impending saccade. This suggest that an interesting extensions of our work could be to investigate how a task such as motion discrimination is affected by external noise, and use this to determine whether there are motion-specific mechanisms of saccadic suppression.

\section{Implementation}

If a simple, stimulus-independent gain change suffices to explain active saccadic suppression, it is tempting to conclude that the mechanism may be implemented at the earliest stages of the visual system. Indeed, it has been argued on the basis of behavioral data that suppression takes place in the lateral geniculate nucleus (LGN) (Burr et al., 1994; Thilo et al., 2004). Our earlier studies, however, show that suppression in one eye is modulated by the luminance in the other eye; such an interaction requires the involvement of the binocular neurons of visual cortex (Chahine and Krekelberg, 2009). Moreover, we have shown that information about the orientation of a perisaccadic visual stimulus survives saccadic omission (Watson and Krekelberg, 2009). This implies that even stimuli that are completely suppressed (i.e., omitted from awareness) are processed by the orientation selective neurons of visual cortex. This is inconsistent with a suppression mechanism that resides entirely in the LGN.

Reduced perisaccadic visual responses have been observed in many areas, using single cell recordings (Reppas et al., 2002; Thiele et al., 2002; Royal et al., 2006; Ibbotson et al., 2008; Kagan et al., 2008; Bremmer et al., 2009; Cloherty et al., 2010; Saul, 2010) as well as functional magnetic resonance imaging (fMRI) (Kleiser et al., 2004; Sylvester and Rees, 2006; Vallines and Greenlee, 2006). Although these reduced responses are consistent with gain reduction and their time course matches the overall pattern of saccadic suppression (Vallines and Greenlee, 2006; Bremmer et al., 2009), the perisaccadic response changes observed in these studies often involve more than just a reduction in gain. Our work suggests that these complex response changes are not related to what we call the active component of saccadic suppression, leaving open the possibility that they are, however, part of backward-masking mechanisms of saccadic omission (Ibbotson and Cloherty, 2009).

Our behavioral data, however, make a more specific prediction: the gain reduction underlying active saccadic suppression should be stimulus-independent. To our knowledge, there have been no physiological experiments in which stimulus contrast of 
perisaccadic stimuli was varied systematically. If, however, the gain reduction is truly stimulus-independent, it should also be observable as a perisaccadic reduction of the neural response in total darkness. In the medial temporal and ventral intraparietal areas of posterior parietal cortex, neural activity is only reduced in the presence of clear visual stimulation (Bremmer et al., 2009). In the medial superior temporal (MST) area, however, average population activity before the saccade starts is similarly reduced in a dimly lit room and when a visual stimulus is present (Bremmer et al., 2009); this is a signature of a stimulus-independent neuronal gain reduction that is consistent with the predictions based on our behavioral data. The findings of Cloherty et al. (2010) provide further support for this view but also reveal additional complexity; most MST cells show a perisaccadic increase of their spontaneous firing rate (i.e., in near darkness) and a decrease in their response to visual stimuli. Although paradoxical at first sight, both effects could serve to reduce the detectability of a stimulus. In terms of the PTM, the increase in spontaneous firing rate corresponds to an increase in additive internal noise, whereas the reduced visual response corresponds to a decrease of the gain. As discussed above, these parameter changes have equivalent outcomes on signal discriminability.

PET imaging in humans shows a reduction in metabolic activity when saccades are made in complete darkness; Paus et al. (1995) interpreted this as a decrease of excitatory neurotransmission. More recent fMRI studies focusing on primary visual cortex (V1) and LGN report an increased blood oxygenation leveldependent (BOLD) signal when saccades are made in pure darkness, but a decreased BOLD signal in the presence of visual stimulation (Sylvester et al., 2005). These opposing effects could be similar to what Cloherty et al. (2010) reported for MST neurons, and both are compatible with the predictions of the PTM model.

The investigation of saccadic suppression has a long history in both the physiological and the behavioral literature, but a direct, quantitative link between a perisaccadic neural and behavioral change has been elusive (for review, see Wurtz, 2008). In our view, experiments based on the formal methods of equivalent noise analysis and combining electrophysiological or functional imaging methods with behavioral report could provide an important step forward in our understanding of visual processing during eye movements.

\section{References}

Akaike H (1974) A new look at the statistical model identification. IEEE Trans Automat Contr 19:716-723.

Binda P, Cicchini GM, Burr DC, Morrone MC (2009) Spatiotemporal distortions of visual perception at the time of saccades. J Neurosci 29:13147-13157.

Bremmer F, Kubischik M, Hoffmann KP, Krekelberg B (2009) Neural dynamics of saccadic suppression. J Neurosci 29:12374-12383.

Burnham KP, Anderson DR (2002) Model selection and multimodel inference: a practical information-theoretic approach, 2nd Edition. New York: Springer.
Burr DC, Holt J, Johnstone JR, Ross J (1982) Selective depression of motion sensitivity during saccades. J Physiol 333:1-15.

Burr DC, Morrone MC, Ross J (1994) Selective suppression of the magnocellular visual pathway during saccadic eye movements. Nature 371:511-513.

Carandini M, Heeger DJ (1994) Summation and division by neurons in primate visual cortex. Science 264:1333-1336.

Carandini M, Heeger DJ, Movshon JA (1997) Linearity and normalization in simple cells of the macaque primary visual cortex. J Neurosci 17:86218644.

Chahine G, Krekelberg B (2009) Cortical contributions to saccadic suppression. PLoS One 4:e6900.

Cloherty SL, Mustari MJ, Rosa MG, Ibbotson MR (2010) Effects of saccades on visual processing in primate MSTd. Vision Res 50:2683-2691.

Diamond MR, Ross J, Morrone MC (2000) Extraretinal control of saccadic suppression. J Neurosci 20:3449-3455.

Greenhouse DS, Cohn TE (1991) Saccadic suppression and stimulus uncertainty. J Opt Soc Am A 8:587-595.

Heeger DJ (1992) Normalization of cell responses in cat striate cortex. Vis Neurosci 9:181-197.

Ibbotson MR, Cloherty SL (2009) Visual perception: saccadic omissionsuppression or temporal masking? Curr Biol 19:R493-R496.

Ibbotson MR, Crowder NA, Cloherty SL, Price NS, Mustari MJ (2008) Saccadic modulation of neural responses: possible roles in saccadic suppression, enhancement, and time compression. J Neurosci 28:10952-10960.

Kagan I, Gur M, Snodderly DM (2008) Saccades and drifts differentially modulate neuronal activity in V1: effects of retinal image motion, position, and extraretinal influences. J Vis 8:1-25.

Kleiser R, Seitz RJ, Krekelberg B (2004) Neural correlates of saccadic suppression in humans. Curr Biol 14:386-390.

Lu ZL, Dosher BA (1998) External noise distinguishes attention mechanisms. Vision Res 38:1183-1198.

Paus T, Marrett S, Worsley KJ, Evans AC (1995) Extraretinal modulation of cerebral blood flow in the human visual cortex: implications for saccadic suppression. J Neurophysiol 74:2179-2183.

Reppas JB, Usrey WM, Reid RC (2002) Saccadic eye movements modulate visual responses in the lateral geniculate nucleus. Neuron 35:961-974.

Royal DW, Sáry G, Schall JD, Casagrande VA (2006) Correlates of motor planning and postsaccadic fixation in the macaque monkey lateral geniculate nucleus. Exp Brain Res 168:62-75.

Saul AB (2010) Effects of fixational saccades on response timing in macaque lateral geniculate nucleus. Vis Neurosci 27:171-181.

Sylvester R, Rees G (2006) Extraretinal saccadic signals in human LGN and early retinotopic cortex. Neuroimage 30:214-219.

Sylvester R, Haynes JD, Rees G (2005) Saccades differentially modulate human LGN and V1 responses in the presence and absence of visual stimulation. Curr Biol 15:37-41.

Thiele A, Henning P, Kubischik M, Hoffmann KP (2002) Neural mechanisms of saccadic suppression. Science 295:2460-2462.

Thilo KV, Santoro L, Walsh V, Blakemore C (2004) The site of saccadic suppression. Nat Neurosci 7:13-14.

Vallines I, Greenlee MW (2006) Saccadic suppression of retinotopically localized blood oxygen level-dependent responses in human primary visual area V1. J Neurosci 26:5965-5969.

Watson TL, Krekelberg B (2009) The relationship between saccadic suppression and perceptual stability. Curr Biol 19:1040-1043.

Wichmann FA, Hill NJ (2001) The psychometric function. I. Fitting, sampling, and goodness of fit. Percept Psychophys 63:1293-1313.

Wurtz RH (2008) Neuronal mechanisms of visual stability. Vision Res 48: 2070-2089. 